\title{
A REMARK ON FINITELY GENERATED MODULES, II.
}

\author{
TADASI NAKAYAMA
}

The following small remark on the relationship of the (quasi-)regularity and the zero-divisor property may be more or less known but does not seem to the writer to have been explicitly stated in a literature.

Proposition 1. Let $\mathfrak{r}$ be a right-ideal in a ring $R$. If and only if $\mathfrak{r}$ is not contained in the radical $N$ of $R$ (or, what is equivalent, $\mathfrak{r}$ is not quasi-regular), $R$ can be imbedded into a ring $S$ with unit element 1 such that there is an element $a$ in $\mathfrak{r}$ for which $1-a$ is a right zero-divisor in $S$.

Proof. The "only if" part is clear. For, if $\mathfrak{r} \subseteq N$ then every element $a$ in $\mathfrak{r}$ is quasi-regular and $1-a$ is regular. To prove the “if” part, let $\mathfrak{r} \neq N$ and let $\mathfrak{m}$ be a non-zero cyclic $R$-right-module with a generator $u_{0}$ such that $\mathfrak{m}=u_{0} \mathfrak{r}$; for the existence of $m$ cf. (the second half of) $V$ in our first note. ${ }^{1)}$ Let $R^{*}$ be the ring $R \oplus Z$ ( $Z$ being the ring of rational integers) in which $x 1=1 x=x$ for every $x \in R$. $m$ may be considered, in natural manner, as a right-module of $R$.* In the module $\mathrm{m} \oplus R^{*}$ we introduce distributive multiplication by

$$
\mathfrak{m}^{2}=0, \quad R \mathfrak{m}=0, \quad 1 u=u \quad(u \in \mathfrak{m}) ;
$$

the product of two elements in $R^{*}$ and the product of an element in $m$ and an element of $R$, in this order, are defined as they already are. The multiplication is associative, and $\mathfrak{m} \oplus R^{*}$ becomes a ring, which we want to denote by S. Now, since $u_{0} \in \mathfrak{r}=u_{0} \mathfrak{r}$, there is an element $a$ in $\mathfrak{r}$ such that $u_{0}=u_{0} a$. In $S$ we have $u_{0}(1-a)=u_{0}-u_{0} a=0$. So $1-a$ is a right zero-divisor in $S$. Our proposition is thus proved.

We have analogously also

Proposition 2. Let $a$ be an element of a ring $R$. If, and only if, $a$ is not right quasi-regular (in $R$ ), we can imbed $R$ into a ring $S$ with a unit element 1 such that $1-a$ is a right zero-divisor in $S$.

Received December 30, 1954.

1) A remark on finitely generated modules, Nagoya Math. J. 3 (1951), 139-140. Thus, we have merely to consider $\mathfrak{m}=R / \mathfrak{b}$ with a maximal right-ideal $\mathfrak{B}$ with left modulo-unit in which $\mathfrak{r}$ is not contained. 
Proof. The "only if" part is again clear, since if $a$ is right quasi-regular in $R$ then $a$ is so in any extension $S$ of $R$. Let, to prove the converse, $a$ be a right non-quasi-regular element in $R$. Then the right-ideal $\mathfrak{r}=\{x-a x \mid x \in R\}$ does not coincide with $R$. Put $\mathrm{m}=R / \mathrm{r}$. Let $R^{*}$ be as in the proof to the preceding proposition. We consider the module $m \oplus R^{*}$ with our present $\mathrm{m}$, and introduce in it a multiplication just in the same manner in the proof to the preceding proposition, $\mathrm{m}^{2}=0, R \mathrm{~m}=0$ and $1 u=u(u \in \mathrm{m})$. Denote the ring so obtained again by $S$. Let $u_{0}$ be the residue-class of $a$ modulo $\mathfrak{r} ; u_{0} \in \mathfrak{m}$ and $u_{0} \neq 0$. Then $u_{0}(1-a)=u_{0}-u_{0} a=0$, since $a-a^{2} \in \mathfrak{r}$. Hence $1-a$ is a right zero-divisor in $S$. This proves our proposition.

The motivation and the relationship to our first note, ${ }^{2)}$ of the above considerations, are the following, besides that we have referred to it. In our first note we generalized namely a theorem of Azumaya and combined the theorem thus obtained with the Jacobson-Kaplansky theory of radicals to produce several propositions which may be summarized as follows: Let $\{\mathfrak{r}\}$ be a certain family of right-ideals in a ring $R$. The following properties of $\{\mathfrak{r}\}$ imply each other:

(A) For every maximal right-ideal $\mathfrak{r}_{0}$ with left modulo-unit of $R$, there exists (at least) a right-ideal $\mathfrak{r}$ in the family $\{\mathfrak{r}\}$ such that $\mathfrak{r} \subseteq \mathfrak{r}_{0}$;

(B) If $\mathrm{m}$ is a finitely generated right-module of $R$ such that $\mathrm{m}=\mathrm{m} R$ and $\mathfrak{m}=u_{1} \mathfrak{r}+\ldots+u_{n} \mathfrak{r}$ for every finite generating system $u_{1}, \ldots, u_{n}$ of $m$ and for every $\mathfrak{r} \in\{\mathfrak{r}\}$, then $\mathrm{m}=0^{3)}$.

2) S. foot note 1 ).

3) The line "the family of right-ideals of III," before the proposition $V$ of the first note, should read "the family of all maximal right-ideals with modulo-unit."

Further, the writer was perhaps too hasty when he wrote, in the proof to the proposition $V$ there, that the implication of (B) from (A) was "evident." The "evident" needs an explanation. It is indeed evident if we take into account (the proposition 1 there and) the fact that for every maximal right-ideal $\mathrm{r}^{*}$ of the ring $R^{*}=R \oplus \mathrm{Z}$ as above (and as there) the intersection $x^{*} \cap R$ is either $R$ itself or a maximal right-ideal of $R$ with left modulo-unit; this fact can readily be seen from that if $\mathfrak{r}^{*} \cup R \neq R$ then $x^{*}+R=R^{*}$ whence there is an element $c \in R$ with $1-c \in \mathrm{r}^{*}$ (whence $x \equiv c x \bmod \mathrm{r}^{*} \cap R$ for all $x \in R$ ).

However, what is perhaps better is to prove the implication directly, and the proof is merely to repeat the argument of our proof to the proposition 1 of the first note. Thus, assume (A). From $\mathfrak{m}=\mathfrak{m} R$ we have $\mathfrak{m}=u_{1} R+\ldots+u_{n} R$, for any generating system $u_{1}$ $\ldots, u_{n}$ of $R$. So $u_{1}$ can be expressed in a form $u_{1}=u_{1} c_{1}+\ldots+u_{n} c_{n}\left(c_{i} \in R\right)$. Let $x_{1}$ be the right-ideal of $R$ consisting of elements $x$ such that $u_{1} x \in u_{2} R+\ldots+u_{n} R$; in case $m=1$ the void sum in the right-hand side stands for 0 . As $u_{1} x=u_{1} c_{1} x+u_{2} c_{2} x+\ldots+u_{n} c_{n} x$ whence $x-c_{1} x \in \mathfrak{r}_{1}$, for any element $x$ of $R, c_{1}$ is a left modulo-unit for $\mathfrak{r}_{1}$. Suppose here $c_{1} \notin \mathrm{r}_{1}$, i.e. $\mathrm{x}_{1} \neq R$. Then there is a maximal right-ideal $\mathfrak{x}_{0}$ of $R$ containing $\mathfrak{r}_{1}$, which evidently possesses $c_{1}$ as a left modulo-unit. By our assumption we have $\mathfrak{m}=\boldsymbol{u}_{1} \mathrm{x}_{0}+\ldots+\boldsymbol{u}_{n} \mathrm{x}_{0}$ whence much the more $\mathrm{m}=u_{1} \mathrm{x}_{0}+u_{2} R+\ldots+u_{n} R$. Expressing $u_{1} c_{1}$ accordingly in a form $u_{1} c_{1}=u_{1} a+\ldots$ with $a \in \mathfrak{x}_{0}$, we have $c_{1}-a \in \mathfrak{r}_{1}\left(a \in \mathfrak{r}_{0}\right)$. This is however a contradiction, since $\mathrm{r}_{1} \leqq \mathrm{x}_{n}, c_{1} \notin \mathrm{x}_{0}$. Thus necessarily $\mathrm{x}_{1}=R$, or, what is the same, $u_{1} R \subseteq u_{2} R+\ldots+u_{n} R$. Now our assertion $m=0$ can be obtained by an easy induction on the minimum number of generating elements. 
(We may restrict ourselves in (B) to cyclic modules and their single generators; cf. the end of the first note.)

The two-sided analogy of this is the following fact, due essentially to Jacobson and Kaplansky (cf. II, III of the first note): Let $N$ be the radical of a ring $R$. The following properties concerning a (two-sided) ideal $M$ are equivalent to each other: $\left(A_{0}\right) M \cong N ;\left(B_{0}\right)$ If $\mathrm{m}$ is a finitely generated rightmodule of $R$ with $\mathrm{m}=\mathrm{m} M$, then necessarily $\mathrm{m}=0$.

The implication $\left(A_{0}\right) \Rightarrow\left(B_{0}\right)$ may be used in proving another theorem of Jacobson that the radical of the matrix ring $R$ (contains and in fact) is $N$ (and indeed a somewhat more general theorem) as Azumaya observes. It is also true that we can prove the implication $\left(A_{0}\right) \Rightarrow\left(B_{0}\right)$ by means of the last matrix ring theorem. For if a right-module $m$ is generated by $u_{1}, \ldots, u_{n}$ and satisfies $\mathfrak{m}=\mathfrak{m} N$, then there are $n$ elements $a_{i j}$ in $N$ such that $\left(u_{1}, \ldots, u_{n}\right)$ $=\left(u_{1}, \ldots, u_{n}\right)\left(a_{i j}\right)$, or $\left(u_{1}, \ldots, u_{n}\right)\left(I-\left(a_{i j}\right)\right)=0$, where $I$ is the unit matrix of degree $n$ considered as the unit element of the matrix ring $\left(R^{*}\right)_{n}$ over the ring $R^{*}$ obtained from $R$ by the adjunction of a unit element 1 . If we know that ${ }^{4)} N_{n}$ is contained in the radical of $\left(R^{*}\right)_{n}$, then we can conclude that $\left(I-\left(a_{i j}\right)\right.$ is regular in $\left(R^{*}\right)_{n}$ whence $)\left(u_{1}, \ldots, u_{n}\right)=0$.

This argument may be modified to show the following fact: Let $n$ be a natural number. Let $M$ be an ideal of $R$. Suppose that, for every set of $n^{2}$ elements $a_{i j}$ of $M$, the element $I-\left(a_{i j}\right)$ of $\left(R^{*}\right)_{n}$ is a right non-zero-divisor in $\left(R^{*}\right)_{n}$. Then, if $\mathrm{m}$ is a right-ideal in $R$ generated by some $n$ elements and satisfies $\mathfrak{m}=\mathfrak{m} M$, then necessarily $\mathfrak{m}=0$.

These considerations seem to show the necessity of clarifying the relationship of the (quasi-)regularity and the non-zero-divisor property, and our answer is what we proved above.

Nagoya University and University of Hamburg

4) Observe that $N$ is contained in (and coincides with, in fact) the radical of $R^{*}$. 